\title{
On the ability of the Generalized Continuum Transport Model to properly capture dispersion
}

\author{
Leonid Vasilyev and Florin Adrian Radu
}

\begin{abstract}
The ability of the Generalized Continuum Transport model to describe dispersion is studied through the comparison of the breakthrough curves with an analytical solution of the linear advection-dispersion Equation. First, a velocity distribution due to Taylor dispersion in a capillary tube is related to the dispersion coefficient of the advectiondispersion equation. The same distribution is applied to the Generalized Continuum Transport model, where the dispersive flux term is not included as the term proportional to the concentration gradient. In the second stage the velocity distribution is obtained from the transition probability introduced through the Continuous Time Random Walk approach. The approaches support the idea that the Generalized Continuum Transport model captures velocity variations naturally through the parameter space. The results confirm that a proper selection of the parameter space, including its size, leads to more physical transport description as well as accurate quantification.
\end{abstract}

\section{Nomenclature}
ADE Advection-Dispersion Equation
BTC Breakthrough curve
CTRW Continuous Time Random Walk model
GCT Generalized Continuum Transport model
TPL Truncated Power Law for the CTRW model

Key Words: Generalized Continuum Transport, Fluid Flow Models, Dispersion, Velocity Distribution, Continuous Time Random Walk, Power Law

Received: December, 2016.

Revised: March, 2017.

Accepted: May, 2017. 


\section{Introduction}

Dispersion phenomena in flow have always attracted attention of scientists and engineers $[1,2,3,4,7,5,6]$. Investigations of dispersion have addressed a broad range of scales starting from the pore scale (Taylor dispersion $[1,2])$ and moving towards larger scales (distribution of pore conductivities, variation in pore matrix permeabilities, etc. $[7,8,9])$.

The linear Advection-Dispersion Equation with constant coefficients (ADE) is widely used for solute transport quantification despite the reported limitations $[11,12,13]$. In the classical ADE formulation all transport variations are summarized in a single dispersion coefficient, which represents neither the nature of those variations nor the scale. Many attempts have been made in order to improve ADE or provide a better transport quantification. Introduction of space- and time-dependent dispersion coefficient leads to a better description of the corresponding processes [14, 15]. Numerous stochastical approaches such as Continuous Time Random Walk (CTRW) [8], Global Random Walk $[16,17]$ and stochastic ADE [18] aimed to capture non-Fickian and memory effects. Multiple porosity and multi-permeability models have improved quantification of transport in porous networks with local heterogeneities and trapping, non-Fickian on a large scale [7, 19].

Generalized Continuum Transport approach (GCT) has been introduced in [20] in order to provide a model, which is continuous in both space and time, provides a better quantification of anomalous effects and has existing models as a limit or as a subset. Despite of this statement, GCT does not limit directly to ADE, where the dispersive flux is proportional to the concentration gradient. It is proposed that the velocity variations should be captured by introducing the velocity distribution over the media together with the mass transfer between them. Adaptive computations based on GCT were proposed in $[10]$.

In this study we implement the velocity variations in a pore channel into the GCT model. The velocity distribution is derived from two formulations: from the velocity profile due to Taylor dispersion and the transition probability distribution of the CTRW approach. The velocity distributions are applied to GCT, where the mass transfer is calculated by fitting the solution of GCT to the solution of ADE obtained under the same conditions. Accordingly, we demonstrate the ability of the GCT model to quantify the velocity variations in a more natural manner, that is advantageous for analysis of the flow dispersion mechanisms.

The article is organized as follows. At first, the velocity distribution due to Taylor dispersion in a capillary channel is addressed in Section 2. The velocity distribution is applied to the GCT model and the mass transfer term 


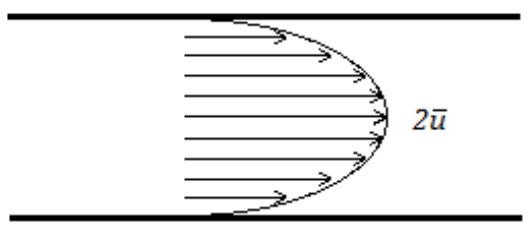

Figure 1: Parabolic velocity profile in a capillary tube

is obtained during the curve fitting. The same assumptions are used in order to derive the coefficients for ADE. Consequently, the solution of GCT is compared with the analytical solution of ADE. In Section 3 the velocity distribution is obtained using the CTRW approach. Same as in Section 2, the solutions of both GCT and CTRW are compared. The results are discussed in Section 4.

\section{Velocity Distribution Due to Taylor Dispersion}

When a fluid flows through a capillary tube, a velocity profile develops due to the wall friction. Thus, the fluid is dispersed along the axis associated with the mean flow direction. This phenomenon has been discussed and quantified in [1] and is called Taylor dispersion. It has also been shown that the velocity profile in a circular pipe is parabolic and can be expressed as $[1,2]$

$$
u(r)=2 \bar{u}\left(1-\frac{r^{2}}{a^{2}}\right),
$$

where $r$ is the distance from the tube center, $\bar{u}$ is the average velocity and $a$ is the radius of the channel (Fig. 1). The effect of Taylor dispersion is compensated by the presence of molecular diffusion which mixes the fluid moving with different velocities across the mean flow direction.

On average, the effect of Taylor dispersion can be expressed by the dispersion coefficient [1]

$$
D_{T}=\frac{\bar{u}^{2} a^{2}}{48 \cdot D_{m o l}} .
$$

Thus, the effective dispersion coefficient becomes

$$
D_{\text {eff }}=D_{m o l}+D_{T},
$$

and the average transport along the capillary can be quantified by the one- 
dimensional version of $\mathrm{ADE}$

$$
\frac{\partial c}{\partial t}+\bar{u} \frac{\partial c}{\partial x}=D_{e f f} \frac{\partial^{2} c}{\partial x^{2}} .
$$

A typical porous system consists of small capillaries, where Taylor dispersion plays a significant role $[1,2,3]$. It has been emphasized by [9] that including Taylor dispersion is important for accurate modeling of transport in porous networks.

In GCT model, the concentration of the contaminant is determined for each physical point in space and time as well as distributed over the space of parameters $\Omega$. This is similar to the multiple-permeability and multi-rate models, where the concentration is given separately for each continuum. In GCT, the concentration is distributed over a continuous space of transport continua such that

$$
c(\mathbf{x}, t, \omega) \in X \times T \times \Omega,
$$

where $X$ denotes a bounded domain in $\mathcal{R}^{d}, d$ being the spatial dimmension and $T$ denotes a finite time interval.

These continua interact with each other through a mass transfer term, which is essentially a function of concentration

$$
N(\mathbf{x}, t, \omega)=\int_{\xi \in \Omega} n(\omega, \xi)(c(\mathbf{x}, t, \omega)-c(\mathbf{x}, t, \xi)) d \xi,
$$

where $n(\omega, \xi)$ is the mass transfer coefficient.

Mass conservation requires that

$$
\int_{\omega \in \Omega} N(\mathbf{x}, t, \omega) d \omega=0,
$$

which follows from the fact that the species do not accumulate but are being redistributed. At the same time, $n(\omega, \xi)$ should be symmetrical because both $n(\omega, \xi)$ and $n(\xi, \omega)$ represent mass transfer between the same two continua.

Following [20], we write GCT in a form

$$
\phi \varphi \frac{\partial}{\partial t} c+\nabla_{\mathbf{x}} \cdot \mathbf{j}=N(\mathbf{x}, t, \omega),
$$

where $\varphi$ is in general a function of $(\mathbf{x}, t, \omega)$, and $\phi$ is a scalar denoting the medium's porosity. Here, $\varphi$ is the probability density to find that species (portion of fluid, moving with flux $\mathbf{j}$ with exchange rate $N$ ) within the medium. 


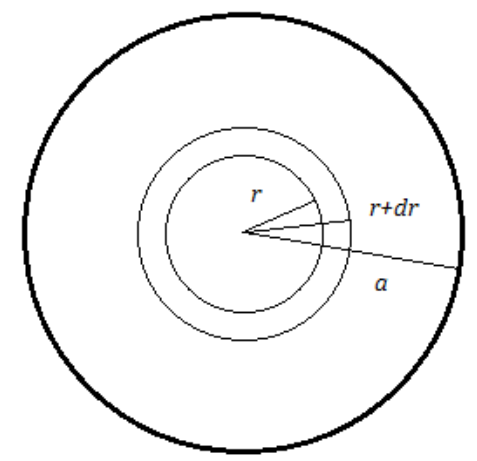

Figure 2: Cross-section of the circular capillary channel

In other words, it is the void space distribution within a porous medium or a fluid part within the channel. Thus, one more requirement should be fulfilled:

$$
\int_{\omega \in \Omega} \varphi(\mathbf{x}, t, \omega) d \omega=1, \forall(\mathbf{x}, t) \in X \times T .
$$

The mass transfer term acts only on $c$ as a function of $\omega$ and accounts for the transport mechanisms in the medium. The fluid flux $\mathbf{j}$ is generally a function of $(c, \mathbf{x}, t, \omega)$, but here we want it to account only for the velocity distribution over $\Omega$. This means that for a homogeneous stationary domain

$$
\mathbf{j}(c, \omega)=\phi \varphi(\omega) \mathbf{u}(\omega) c(\mathbf{x}, t),
$$

where $\mathbf{u}(\omega)$ can be obtained directly from the Taylor velocity distribution.

Consider a circular channel, where the velocity profile is parabolic, and consider a part of the fluid which has the longitudinal velocities lower than $u$. Obviously, these velocities can be found within the $\operatorname{ring}$ with radii $R$ and $a$. The portion of the fluid, moving within the given velocity range, is related to the area of this ring

$$
P(r>R)=\frac{a^{2}-R^{2}}{a^{2}} .
$$

Substituting $r$ with $u$ using the relation (1), we get

$$
P(u<U)=U / 2 \bar{u} \text {. }
$$

Thus, the velocity's density function is of the form

$$
\varphi(u)=\frac{d P}{d U}=1 / 2 \bar{u} .
$$


which corresponds to the uniform probability distribution. This velocity distribution can be used directly in (8) and represents the effect of Taylor dispersion, while the mass transfer between the fluid portions acts similar to molecular diffusion.

For analysis, the capillary dimensions are given in Table 1 and are chosen such, that they represent a typical pore channel similar to those in [9].

\begin{tabular}{|l|c|}
\hline Channel radius & $0.3 \mathrm{~mm}$ \\
\hline Molecular diffusion coefficient & $10^{-3} \mathrm{~mm}^{2} / \mathrm{s}$ \\
\hline Effective dispersion coefficient & $3.17 \mathrm{~mm}^{2} / \mathrm{s}$ \\
\hline Drift velocity & $1.3 \mathrm{~mm} / \mathrm{s}$ \\
\hline
\end{tabular}

Table 1: Parameters of the pore channel

The effective dispersion coefficient is calculated using the relations (2) and (3), and it is 3 orders of magnitude larger than the molecular diffusion coefficient. The velocity distribution is discretized in order to resolve the GCT equation numerically. Thus in case of a long capillary channel (8) becomes

$$
\varphi(\omega) \frac{\partial c(\omega)}{\partial t}+\varphi(\omega) \bar{u}(\omega) \frac{\partial c(\omega)}{\partial x}=\sum_{\xi \in \Omega} n(\omega, \xi)(c(\omega)-c(\xi)),
$$

where the mass transfer coefficient $n(\omega, \xi)$ will be numerically fitted. Here and below we omit writing the variables $\mathbf{x}, t$ for the solute concentration, though it is, of course, distributed over space and time. The porosity coefficient is not applicable for the channel volume, and therefore $\phi=1$.

In the discretized form of GCT $(14) \varphi(\omega)$ is exactly the portion of the fluid, traveling with the average velocity $u(\omega)$, given by

$$
\varphi(\omega)=\varphi(u(\omega)<u<u(\omega)+\Delta u)=\int_{u(\omega)}^{u(\omega)+\Delta u} \frac{1}{2 \bar{u}} d u .
$$

Thus, $\Omega$ here corresponds to the discretization of the capillary channel; hence, the velocity profile in the channel.

The velocity distribution is obtained directly from (13) and applied to (14). At the same time, the reference BTCs are obtained through an analytical solution of ADE in case of a semi-infinite domain with constant boundary condition and zero (no solute) initial state

$$
\begin{aligned}
& c(x=0, t)=1, t \in T \\
& c(x, t=0)=0, x \in X .
\end{aligned}
$$


The analytical solution of ADE for such system is known to be [21]

$$
c_{A D E}(x, t)=\operatorname{erfc}\left(\frac{x-\bar{u} t}{\sqrt{2 D_{e f f} t}}\right),
$$

where $\operatorname{erfc}(x)$ is the cumulative error function defined as

$$
\operatorname{erfc}(x)=\frac{2}{\sqrt{\pi}} \int_{x}^{\infty} e^{-z^{2}} d z .
$$

Consequently, the reference BTCs are fitted with the solution of (14) in order to obtain the matrix of mass transfer coefficients $n(\omega, \xi)$. The parameter of the analysis is the size of $\Omega$, which represents the discretization of the velocity distribution across the channel.

Discretization of the velocity distribution assumes that the velocity of the fluid between the given radii is treated as constant. The thickness of the ring (Fig. 2) corresponds to the idea that molecular diffusion counteracts Taylor dispersion. Larger $d x$ results in averaging across the channel which represents mixing, similar to molecular diffusion (same as in (eq:Tdisp)). Therefore, the minimal size of the ring is directly related to the molecular diffusion coefficient.

The solution of (14) is obtained using the first order upwind finite-difference method in space and backward Euler in time. For a discussion on the numerical diffusion for related discretization schemes we refer to [27]. The equation (14) is rewritten in a form

$$
\begin{aligned}
\varphi(\omega) \frac{c_{k}^{n}(\omega)-c_{k}^{n-1}(\omega)}{\Delta t}= & -\varphi(\omega) \bar{u}(\omega) \frac{c_{k}^{n}(\omega)-c_{k-1}^{n}(\omega)}{\Delta x}+ \\
& +\sum_{\xi \in \Omega} n(\omega, \xi)\left(c_{k}^{n}(\omega)-c_{k}^{n}(\xi)\right),
\end{aligned}
$$

enclosed with initial conditions (uncontaminated domain)

$$
\forall \omega \in \Omega, \quad c(x, t=0, \omega)=0, \quad x \in X
$$

and boundary conditions (full contamination at the inlet)

$$
\forall \omega \in \Omega, \quad c(x=0, t, \omega)=1, \quad t \in T .
$$

The average concentration $\bar{c}(x, t)$ is calculated from the weighted sum:

$$
\bar{c}(x, t)=\sum_{\omega \in \Omega} \varphi(\omega) c(\omega) .
$$


Hence, the GCT model in the discrete form (when $\Omega$ is a finite set) transforms into the multiple continua model (14), and (19) is simply a system of algebraic equations. The velocity distribution, discussed above, provides directly the vectors $\varphi(\omega)$ and $\bar{u}(\omega)$, while the mass transfer rate matrix $n(\omega, \xi)$ is found by fitting the corresponding BTCs. The fitting of the curves and derivation of the model unknowns (mass transfer rates) is performed using the least squares method in combination with Nelder-Mead optimization method [23] in order to minimize the fitting error of a multivariate function. This method is widely used due to its robustness as it is derivative free. The minimum of a multivariate function is estimated based on the function values at a given point sequence calculated by the algorithm. Here we minimize the fitting error, which is defined as

$$
e r r=\sum_{x \in X_{p}} \frac{1}{N} \sum_{i=1}^{N} \sqrt{\left(c_{A D E}\left(x, t_{i}\right)-\bar{c}\left(x, t_{i}\right)\right)^{2}},
$$

where $X_{p}$ is the subset of $X$, and it is the finite set of observation points. The concentration is observed at some time points $t_{i}$, where $N$ is the number of those points. It is logical to define the fitting criterion to be the least squares, as the BTCs are usually smooth and do not experience any local spikes. The fitting process is suggested to be completed when the error tolerance reached $10^{-10}$, with the parameter tolerance set to $10^{-6}$. Thus, the parameters are found when those criteria have been fulfilled.

The results of the curve fitting are presented on Fig. 3: fitting error is plotted against the size of the parameter spase $\Omega$ which corresponds to the velocity profile discretization. It is obvious, that a poor discretization of the velocity distribution (smaller size of $\Omega$ ) leads to higher fitting error and improper representation of the processes in the considered pore channel. On Fig. 4 the solution of $\mathrm{ADE}$ (the reference BTC) is plotted together with the fitted solution of GCT.

\section{Truncated Power Law for Transition Time Distribu- tion}

Another interesting source of the velocity distribution is the Truncated Power Law (TPL) for the Continuous Time Random Walk model (CTRW) [8]. In CTRW the flow domain is discretized, and a probability $\psi(\mathbf{s}, t)$ of the particle displacement $\mathbf{s}$ per time $t$ is introduced. The probability per time for a particle 


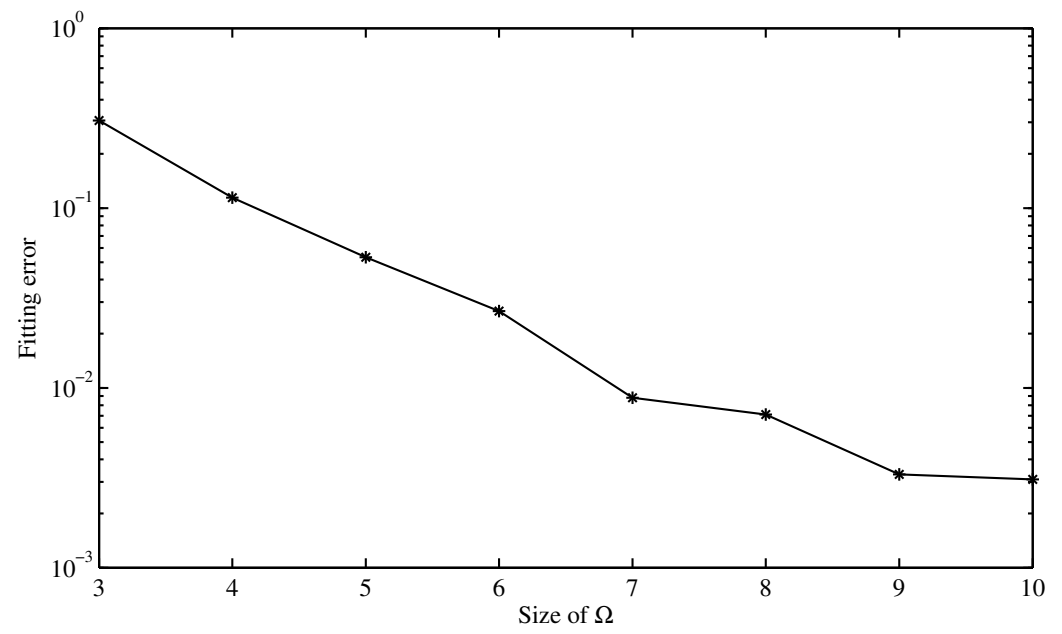

Figure 3: Error of fitting the BTCs of GCT with Taylor based velocity distribution to the solution of ADE.

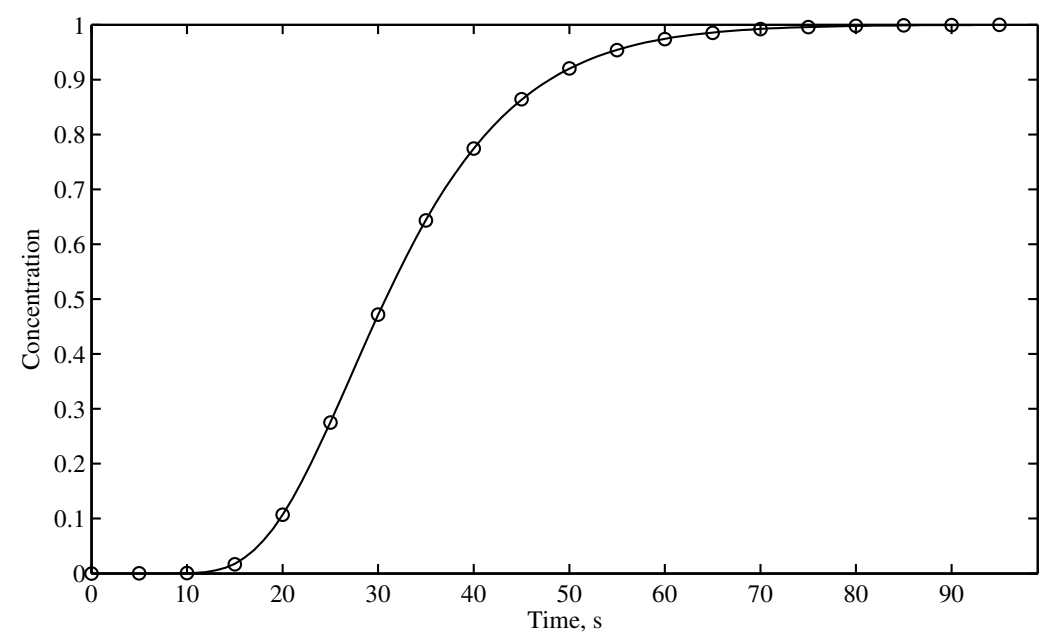

Figure 4: Solution of ADE (solid line) plotted together with the solution of GCT. Solution of GCT with Taylor based velocity distribution coincide completely with the solution of GCT with the TPL based distribution. 
to arrive at site $\mathbf{s}$ at time $t$ is given by

$$
R(\mathbf{s}, t)=\sum_{\mathbf{s}^{\prime}} \int_{0}^{t} \psi\left(\mathbf{s}-\mathbf{s}^{\prime}, t-t^{\prime}\right) R\left(s^{\prime}, t^{\prime}\right) d t^{\prime} .
$$

Thus, (24) leads to non-local processes, whose current state depends on the set of the previous states $R\left(\mathbf{s}^{\prime}, t^{\prime}\right)$. It is essential that $\psi\left(\mathbf{s}-\mathbf{s}^{\prime}, t-t^{\prime}\right)$ is defined for a displacement $\left(\mathbf{s}-\mathbf{s}^{\prime}\right)$ with a difference of arrival times $\left(t-t^{\prime}\right)$ and does not depend on the process history. The probability of the particle to leave the site after time $t$ is given by

$$
\psi(t)=\sum_{\mathbf{s}} \psi(\mathbf{s}, t)
$$

CTRW approach is completely equivalent to the Generalized Master Equation $[22,24]$ with the correspondence

$$
c(\mathbf{s}, t)=\int_{0}^{t} \Psi\left(t-t^{\prime}\right) R\left(\mathbf{s}, t^{\prime}\right) d t^{\prime}
$$

from which the concentration of the contaminant can be calculated. Here, $\Psi(t)$ is the probability of particles to remain at the site

$$
\Psi(t)=1-\int_{0}^{t} \psi\left(t^{\prime}\right) d t^{\prime}
$$

A more complete knowledge about the CTRW approach can be obtained through [8] and references therein.

$\psi(t)$ is the probability density function that accounts for transport nature. A common way to define $\psi(t)$ is the TPL model [25] which accounts for the transition from normal to anomalous (non-Fickian) transport, and thus, is also suitable for our needs:

$$
\psi(t)=\left[t_{1}\left(t_{2} / t_{1}\right)^{-\beta} e^{t_{1} / t_{2}} \Gamma\left(-\beta,\left(t_{1} / t_{2}\right)\right)\right]^{-1} \frac{e^{-t / t_{2}}}{\left(1+t / t_{1}\right)^{1+\beta}},
$$

where $\Gamma$ is the incomplete Gamma-function [26]. TPL model is, therefore, determined by the parameters $\beta, t_{1}$ and $t_{2}$, which can be obtained by fitting the BTCs.

$\psi(t)$ defines the probability of transition at time $t$. In order to assess the velocity distribution we introduce the mean transition time which is defined 


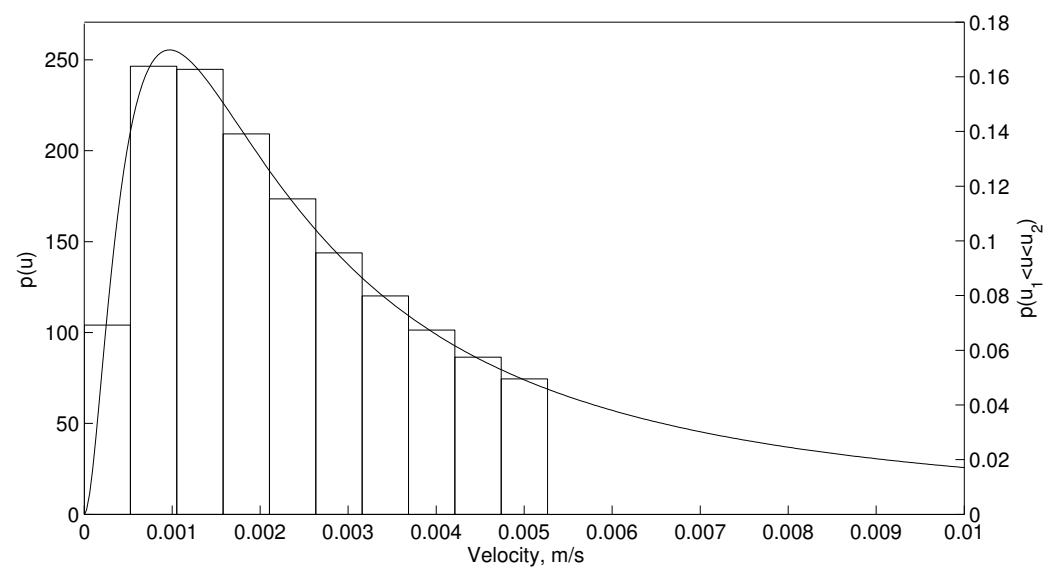

Figure 5: Velocity distribution (solid line, left axis) and its discretized form (ten bins, right axis) obtained by fitting the BTCs to the TPL type CTRW model.

as [25]:

$$
t_{\text {mean }}=-t_{2}\left[\beta+t_{1} / t_{2}-\frac{\left(t_{1} / t_{2}\right)^{-\beta} e^{-t_{1} / t_{2}}}{\Gamma\left(-\beta, t_{1} / t_{2}\right)}\right]
$$

and is related to the drift velocity and the mean displacement as:

$$
\bar{u}=s_{\text {mean }} / t_{\text {mean }} .
$$

Consequently, the velocity distribution is derived by substituting the transition time with the transition velocity $u=s_{\text {mean }} / t$. Here $\psi(u)$ is the cumulative distribution function. The corresponding probability density function can be found from its differential:

$$
\varphi(u) d u=d \psi(u) .
$$

The BTCs, obtained previously, are fitted with the CTRW model with TPL distribution of transition time. Consequently, the distribution is transformed into the velocity distribution which is then applied into the GCT model (14) in discretized form (one discretization example is presented on Fig. 5):

$$
\varphi(u(\omega)<u<u(\omega+d \omega))=\psi(u(\omega))-\psi(u(\omega+d \omega)) .
$$

Same as before, the discretization resolution varied from 3 to 10 and this is the main parameter for fitting the BTCs with the GCT model. It was observed 


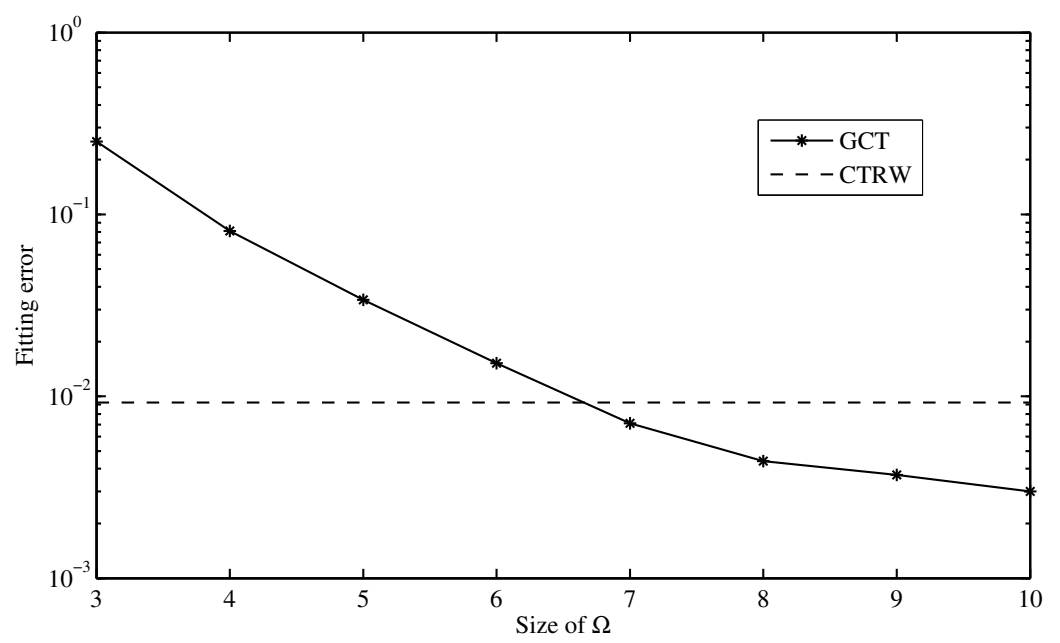

Figure 6: Fitting error of GCT with TPL originated velocity distribution (solid curve). Dashed line represents the fitting of the CTRW model, which was the source of the distribution.

that $\beta>2$, which leads to Fickian type dispersion [8]. This is a consequence of the BTCs' origin, that was obtained from the solution of ADE. The results of the curve fitting are presented on Fig. 6 and Fig. 4. Same as for Taylor velocity distribution, the GCT model demonstrates excellent correspondence when the size of $\Omega$ is sufficiently high. Thus, GCT captures the velocity variations by introducing the corresponding velocity distribution into the model.

\section{Conclusions}

The ability of the GCT model to capture the dispersive transport effects is demonstrated by applying the velocity distributions from two sources: parabolic profile in a channel due to Taylor dispersion and transition time distribution of the CTRW TPL model. The solutions of GCT and ADE are compared in terms of the discretization resolution of velocity distributions. It is essential that the velocity variations are introduced in the model without the second order term, which is in contrast to ADE.

It is remarkable that a proper discretization of the velocity distribution across the pore channel gives good fitting results, while a lower resolution leads to large error. This supports the suggestion that molecular diffusion counteracts the development of the parabolic velocity profile. Hence, intro- 
duction of the larger fluid regions, where velocity is treated as the constant average, seems to lead to stronger mixing in the transversal direction.

The same error vs. resolution tendency appears when the TPL type distribution is applied to the GCT model. The transition time distribution corresponds to Fickian behavior which is reasonable, since the BTCs were obtained directly from the solution of ADE.

Finally, we conclude that flow variations are implemented more naturally in the GCT model through the corresponding distribution of parameters. At the same time, GCT is continuous in both space and time which makes it an interesting tool for investigation of transport mechanisms both numerically and analytically.

\section{References}

[1] G. Taylor, Diffusion and mass transport in tubes, Proc. Phys. Soc. B 67(857), 1954.

[2] R. Aris, On the dispersion of a solute in a fluid flowing through a tube, Proc. Roy Soc. London A 235:67, 1956.

[3] J. Glimm, W.B. Lindquist, F. Pereira, Q. Zhang, A theory of macrodispersion for the scale up problem, Transp. Porous Med. 13: 97, 1993.

[4] N. Suciu, Diffusion in random velocity fields with applications to contaminant transport in groundwater, Adv. Water Resour., 69: 114-133, 2014.

[5] N. Suciu, S. Attinger, F. A. Radu, C. Vamos, J. Vanderborght, H. Vereecken and P. Knabner, Solute transport in aquifers with evolving scale heterogeneity, An. St. Univ. Ovidius Constanta, 23(3): 167-186, 2015 .

[6] N. Suciu, F.A. Radu, S. Attinger, L. Schueler and P. Knabner, A FokkerPlanck approach for probability distributions of species concentrations transported in heterogeneous media, J. Comput. and Appl. Math., 289 (1): 114-133, 2015.

[7] J.P. Gwo, P.M. Jardine, G.V. Wilson, G.T. Yeh: Using a multiregion model to study the effects of advective and diffusive mass transfer on local physical nonequilibrium and solute mobility in a structured soil, Wat. Resour. Res., 32: 561, 1996. 
[8] B. Berkowitz, A. Cortis, M. Dentz, H. Scher, Modeling non-Fickian transport in geological formations as a continuous time random walk, Rev. Geophys., 44: RG2003, 2006.

[9] L. Vasilyev, A. Raoof, J.M. Nordbotten, Effect of Mean Network Coordination Number on Dispersivity Characteristics, Transp. Porous Med. 95: 447-463, 2012.

[10] L. Vasilyev, J.M. Nordbotten, F.A. Radu and K. Kumar, On the properties of the parameter space of generalized continuum transport model for description of fluid flow in porous networks, submitted, 2017.

[11] J.S. Aronofsky, J.P. Heller: A diffusion model to explain mixing of flowing miscible fluids in porous media, Trans. Am. Inst. Min. Metall. Pet. Eng., 210: 345-349, 1957.

[12] A.E. Scheidegger: An evaluation of the accuracy of the diffusivity equation for describing miscible displacement in porous media, Proc. Theory Fluid Flow Porous Media Conf. 2nd: 101-116, 1959.

[13] C. Kennedy, W. Lennox: A stochastic interpretation of the tailing effect in solute transport, Stochastic Environmental Research and Risk Assesment, 15: 325-340, 2001.

[14] D.K. Jaiswal, A. Kumar, R.R. Yadav, Analytical Solution to the OneDimensional Advection-Diffusion Equation with Temporally Dependent Coefficients, Journal of Water Resource and Protection, 3: 76-84, 2011.

[15] N. Suciu, C. Vamos, F.A. Radu, H. Vereecken, P. Knabner, Persistent memory of diffusing particles, Phys. Rev. E 80:061134, 2014.

[16] C. Vamos, N. Suciu, H. Vereecken, Generalized random walk algorithm for the numerical modeling of complex diffusion process, J. Comput. Phys. 186(2): 527-44, 2003.

[17] N. Suciu, C. Vamos, H. Vereecken, P. Knabner, Global random walk simulations for sensitivity and uncertainty analysis of passive transport models, Annals of the Academy of Romanian Scientists, Series on Mathematics and its Applications 3 (1), 2011.

[18] B. Berkowitz, J. Klafter, R. Metzler, H. Scher, Physical pictures of transport in heterogeneous media: Advection dispersion, random-walk, and fractional derivative formulations, Water Resour. Res., 38(10): 1191, 2002 . 
[19] T. Vogel, H.H. Gerke, R. Zhang, M.Th. van Genuchten, Modeling flow and transport in a two-dimensional dual-permeability system with spatially variable hydraulic properties, Journal of Hydrology, 238: 78-89, 2000.

[20] J.M. Nordbotten, L. Vasilyev, On the relationship between multiple porosity models and continuous time random walk, XVIII International Conference on Water Resources CMWR2010, 2010.

[21] M.Th. van Genuchten, W.J. Alves, Analyitical slutions of the onedimensional convective-dispersive solute transport equation. U.S. Department of Agriculture, Technical Bulletin, No. 1661, 1982.

[22] V.M Kenkre, E.W. Montroll, M.F. Shlesinger, Generalized master equations for continuous-time random walks, J. Stat. Phys., 9(1): 45-50, 1973.

[23] J.A. Nelder, R. Mead, A simplex method for function optimization. Comput. J. 7: 308-313, 1965.

[24] M.F. Shlesinger, Asymptotic solutions of continuous-time random walks, J. Stat. Phys., 10(5): 421-434, 1974.

[25] M. Dentz, A. Cortis, H. Scher, B. Berkowitz, Time behavior of solute transport in heterogeneous media: Transition from anomalous to normal transport, Adv. Water Resour., 27: 155-173, 2004.

[26] M. Abramowitz, I. Stegun, Handbook of Mathematical Functions, Dover, Mineola, N.Y., 1970.

[27] F.A. Radu, N. Suciu, J. Hoffmann, A. Vogel, O. Kolditz, C-H. Park and S. Attinger, Accuracy of numerical simulations of contaminant transport in heterogeneous aquifers: a comparative study. Adv. Water Resour., 34 (1): 47-61, 2011.

Leonid Vasilyev,

Department of Mathematics,

University of Bergen,

Postboks 7803, N-5020, Bergen, Norway

Email: leonid.vasilyev@uib.no

Florin Adrian Radu,

Department of Mathematics,

University of Bergen,

Postboks 7803, N-5020, Bergen, Norway

Email: florin.radu@uib.no 